\title{
Self-assemblying Classes of Shapes with a Minimum Number of Tiles, and in Optimal Time ${ }^{\star}$
}

\author{
Florent Becker ${ }^{1}$, Ivan Rapaport ${ }^{2}$, and Éric Rémila ${ }^{1}$ \\ ${ }^{1}$ Laboratoire de l'Informatique du Parallélisme, \\ UMR 5668 CNRS-INRIA-Univ. Lyon 1-ENS Lyon, France \\ \{florent.becker, eric.remila\}@ens-lyon.fr \\ ${ }^{2}$ Departamento de Ingeniería Matemática and \\ Centro de Modelamiento Matemático \\ UMR 2071-CNRS, Universidad de Chile \\ irapapor@dim.uchile.cl
}

\begin{abstract}
In this paper we construct fixed finite tile systems that assemble into particular classes of shapes. Moreover, given an arbitrary $n$, we show how to calculate the tile concentrations in order to ensure that the expected size of the produced shape is $n$. For rectangles and squares our constructions are optimal (with respect to the size of the systems). We also introduce the notion of parallel time, which is a good approximation of the classical asynchronous time. We prove that our tile systems produce the rectangles and squares in linear parallel time (with respect to the diameter). Those results are optimal. Finally, we introduce the class of diamonds. For these shapes we construct a non trivial tile system having also a linear parallel time complexity.
\end{abstract}

\section{Introduction}

The tile assembly model was introduced by Rothemund and Winfree [517. This model, based on the classical one of Wang [6], includes a mechanism of growth (a dynamics) which takes into account global parameters such as the temperature and the tile concentrations.

The individual components are square tiles. These tiles "float" on the two dimensional plane. They can not be rotated. Each side of a tile has a specific "glue". When two tiles collide they stick if their abbuting sides have the same glue and, crucially, if the strength of the glue is "high enough" with respect to the temperature.

The dynamics of such a tile system is modeled as a Markov process. The precise process we consider here was introduced by Adleman et al. [1]. It is, however, a simplification of the reversible version proposed by Winfree [7. Roughly speaking, the higher the concentration of a particular tile the higher the rate at which

\footnotetext{
* This work was supported by programs Ecos-Conicyt, Conicyt "Anillo en Redes", and Fondap on Applied Mathematics.
} 
it is encountered. And, when encountered, the particular tile can eventually be incorporated into the growing structure. At the end of the process, which begins with a "seed" tile placed at the origin of the plane, a given shape $S$ will be produced. Aggarwal et al. 2] proved that the minimal number of tiles that uniquely produces the $m \times n$ rectangle is $\Omega\left(\frac{n^{\frac{1}{m}}}{m}\right)$ if $m \ll n$ and $\Theta\left(\frac{\log n}{\log \log n}\right)$ otherwise. In [1] it is shown that the average time complexity for uniquely producing an $n \times n$ square is $\Theta(n)$.

Of course, besides the assembly time, some other random variables are also relevant. In fact, in this work we focus our attention on the random variable that corresponds to the "size" of the produced shape. We therefore explore a new direction by searching for tile systems producing languages of shapes. We tackle the problem of producing three natural languages of shapes: squares, rectangles and diamonds.

Let us consider, for instance, the class of all squares. In our construction we fix the tile system in such a way that each time we run the Markov chain a (different) square is produced. Let us call $N$ the random variable corresponding to the size of the produced square. If $n$ is a fixed positive integer, then we will show how to calculate the tile concentrations in order to ensure that $\mathbb{E}(N)=n$. To our knowledge, the tile concentrations, a parameter of the original model, has never been seriously considered before. In other words, it is therefore not necessary to construct the tiles (which in fact are model representations of tiny molecules) for each shape we are asked to assemble.

We construct tile systems for rectangles and squares. Each of them turn out to be optimal with respect to its size. On the other hand we introduce the notion of parallel time. It is defined as the time needed to assemble a shape when all possible transitions are performed at once. Our constructions for squares and rectangles are also optimal in terms of the parallel time. In fact we get, with respect to the diameter of the shapes, a linear parallel time. We also show that the parallel time gives lower and upper bounds for the average time of the Markov process.

Finally we introduce the class of diamonds. A first approach gives a tile system with quadratic parallel time complexity (linear with respect to the surface). Nevertheless, by using a "firing squad" approach, we get the optimal linear parallel time complexity (linear with respect to the diameter).

\section{Tile Systems}

A tile system is a 5 -tuple $\mathbf{T}=<T, t_{0}, \tau, g, P>$. Each of these variables is defined in the following.

The set of tiles. $T$ is a finite set of tiles. Each of these tiles is an oriented unit square with the north, east, south and west edges labeled from some alphabet $\Sigma$ of glues (or colors). For each $t \in T$, the labels of its four edges are canonically denoted $\sigma_{N}(t), \sigma_{E}(t), \sigma_{S}(t)$ and $\sigma_{W}(t)$.

The seed. $t_{0} \in T$ is a particular tile known as the seed.

The temperature. $\tau$ is a positive integer called the temperature. 
The strength function. The (glue) strength function $g$ goes from $\Sigma \times \Sigma$ to $\mathbb{N}$. We assume that $g(\alpha, \beta)=0$ for all $\alpha, \beta$ in $\Sigma$ such that $\alpha \neq \beta$. The value $g(\alpha, \alpha)$ is called the strength of $\alpha$. We also assume that the set of glues $\Sigma$ contains a special one, denoted by null, such that for all $\alpha$ in $\Sigma, g(n u l l, \alpha)=0$. The tiles are represented as in Figure 1 the number of lines in front of the glue corresponds to the strength of it. There is one exception to that convention: no lines also mean strength 1.

The concentration. The concentration $P$ associates to each tile $t \in T$ a positive value $P(t)$. The concentration function $P$ satisfies $\Sigma_{t \in T} P(t)=1$.
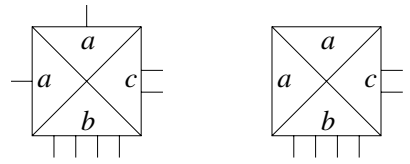

Fig. 1. Two ways of representing the same tile

In order to define the asynchronous and parallel dynamics we need to introduce the following concepts.

T-transitions. A configuration is a map from $\mathbb{Z}^{2}$ to $(T \cup\{$ empty $\})$, where the tile empty is the one having in its four sides the glue null. Let $A$ and $B$ be two configurations. Suppose that there exist $t \in T$ and $(x, y) \in \mathbb{Z}^{2}$ such that $A=B$ except for $(x, y)$ with $A(x, y)=$ empty and $B(x, y)=t$. If also

$$
\begin{gathered}
g\left(\sigma_{E}(t), \sigma_{W}(A(x+1, y))+g\left(\sigma_{W}(t), \sigma_{E}(A(x-1, y))+\right.\right. \\
g\left(\sigma_{N}(t), \sigma_{S}(A(x, y+1))+g\left(\sigma_{S}(t), \sigma_{N}(A(x, y-1)) \geq \tau\right.\right.
\end{gathered}
$$

then we say that the position $(x, y)$ is attachable in $A$, and we write $A \rightarrow_{z} B$ with $z=(t,(x, y))$. We write $A \rightarrow \mathbf{T} B$ when such a $z$ exists. Informally, this means that $B$ can be obtained from $A$ by adding a tile $t$ in such a way that the total strength of the interaction between $A$ and $t$ is at least $\tau$. Let $\rightarrow{ }_{\mathbf{T}}^{*}$ denote the transitive closure of $\rightarrow \mathbf{T}$.

Derived supertiles. The seed configuration, $\Gamma_{t_{0}}$, is the one that satisfies $\Gamma_{t_{0}}$ $(0,0)=t_{0}$ and, for all $(x, y) \neq(0,0), \Gamma_{t_{0}}(x, y)=$ empty. The derived supertiles of the tile system $\mathbf{T}$ are those configurations $X$ such that $\Gamma_{t_{0}} \rightarrow_{\mathbf{T}}^{*} X$.

Production of shapes. A shape is a 4 -connected finite subset of $\mathbb{Z}^{2}$. The shape of a derived supertile $A$ will be denoted by $[A]$ and corresponds to $\{(x, y) \in$ $\mathbb{Z}^{2}: A(x, y) \neq$ empty\}.

\subsection{The Asynchronous Dynamics}

The dynamics of the tile system $\mathbf{T}$ is modeled as a continous time Markov process where the states are in one-to-one correspondence with the derived 
supertiles and the initial state corresponds to the seed coniguration $\Gamma_{t_{0}}$. There is a transition from state $A$ to state $B$ if $A \rightarrow \mathbf{T} B$. If $B$ is obtained from $A$ by adding the tile $t$, then the rate of the transition is $P(t)$. More precisely, it is assumed that the time for the occurence of such a transition follows an exponential law of parameter $P(t)$, and, consequently, the average time necessary to make this transition is $1 / P(t)$.

Suppose that in state $A$ there are $k$ possible transitions to states $B_{1}, \ldots, B_{k}$. And suppose that the transition rates are $P_{1}, \ldots, P_{k}$. Then, the probability to jump to state $B_{i}$ equals $\frac{P_{i}}{P_{1}+\ldots+P_{k}}$. Finally, the time spent in state $A$ follows an exponential law of parameter $P_{1}+\ldots+P_{k}$.

A derived supertile $A$ is called terminal if it is a sink state of the Markov process. In other words, if there is no supertile $B$ such that $A \rightarrow \mathbf{T} B$. The set of shapes produced by the tile system is $S(\mathbf{T})=\{[A]: A$ is terminal $\}$.

Let $\mathcal{C}$ be a set of shapes. We say that the tile system $\mathbf{T}$ uniquely produces the set $\mathcal{C}$ if on one hand $S(\mathbf{T})=\mathcal{C}$ and, on the other hand, the event "the structure grows indefinitely" has probability zero of ocurrence. This notion is a natural generalization of the one of Winfree where the set $\mathcal{C}$ was a singleton.

\subsection{The Parallel Dynamics}

Here, at each step, all possible transitions are performed at once. This parallel dynamics is deterministic. Sometimes it is easy to compute and it allows us to obtain bounds for the assembly time of the asynchronous model.

Let $A$ be a derived supertile, and let Trans be a set of transitions. Trans is a set of independent transitions if, for any $z, z^{\prime} \in$ Trans such that $A \rightarrow_{z} A_{z}$ and $A \rightarrow z^{\prime} A_{z^{\prime}}, z^{\prime}$ is a possible transition from $A_{z}$ and $z$ is a possible transition from $A_{z^{\prime}}$.

There can be several maximal sets of independent transitions from a given supertile. Nevertheless, given a terminal supertile $F$ and a derived supertile $A$, one can define a unique $\| \mathbf{T}$-transition from $A$ to a $A^{\prime}$ such that $F$ can be derived from $A^{\prime}$. This parallel transition is given by taking the set of all attachable positions in $A$, and attaching to each of them the corresponding tile of $F$, when it is already attachable: let Trans $=\left\{t_{1}, \ldots, t_{k}\right\}$ be a maximal set of independent transitions from $A$ which are compatible with $F$. Let $A_{k}$ be such that $A \rightarrow t_{1} A_{1} \ldots A_{k-1} \rightarrow t_{k} A_{k}$. We say that there is a parallel transition between $A$ and $A_{k}$, and we note $A \rightarrow \| \mathbf{T} A_{k}$.

Thus, the sequence of parallel transitions by which $F$ is built is unique.

The parallel time $\pi_{A}$ to assemble a derived supertile $A$ is the number of parallel transitions needed to produce $A$. We will see that the parallel time is closely related to $\tau_{A}$, the expected (asynchronous) assembly time of $A$. The parallel instant of a position $(x, y)$ is the number of the parallel transition that puts a tile at $(x, y)$.

This notion of parallel time is the adequate notion to compute the time efficiency of tilesets, as it is easier to compute than the expected time to complete the Markov process, but is a good approximation. 
Proposition 1. For every tile system $\boldsymbol{T}$ and every production $P$ of $\boldsymbol{T}$, we have $\left(1 / \chi_{\max }\right) \pi_{P} \leq \tau_{P}$, and $\tau_{P}=O\left(\pi_{P} / \chi_{\min }\right)$, where $\chi_{\min }$ and $\chi_{\max }$ are the minimal and maximal tile concentrations. When all the concentrations are $1 / k$, with $k$ the number of tiles in $\boldsymbol{T}$, we have $\tau_{P}=\Theta\left(\pi_{P} k\right)$.

We only give an idea of the proof, which is an extension of the proof of Adleman and Cheng 1 . They stated a version of this prosposition for cases where there is an order of dependency between the positions. But this order does not exist in every case; when it does not exist, we take the order induced by the parallel instants. This order has the properties of fairness that allow us to prove the proposition: any order that is compatible with the assembly contains a chain of length at least $\pi_{p}$.

\section{$3 \quad$ Rectangles and Squares}

Let $m, n$ be positive integers. Let $R_{m, n}=\{(x, y) \mid 0 \leq x<m, 0 \leq y<n\}$ be the rectangle of width $m$ and height $n$. Let us fix the temperature $\tau=2$. With this temperature, by generalyzing the result of [1], it has been proved in [2] that the minimal number of tiles that uniquely produce the rectangle $R_{m, n}$ is $\Omega\left(\frac{n \frac{1}{m}}{m}\right)$ if $m \ll n$ and $\Theta\left(\frac{\log n}{\log \log n}\right)$ otherwise.

Let us fix the set of tiles that appears in Figure 2, Let us consider $t_{S W}$ as the seed. It is easy to notice that this set of tiles uniquely produces rectangles. If $A, B, C$ are arbitrary positive values satisfying $A+B+C=1$, then we fix the concentrations as follows:

$$
\begin{array}{ccc}
P\left(t_{S}\right)=\frac{A(m-2)}{m-1} & P\left(t_{S E}\right)=\frac{A}{m-1} & P\left(t_{W}\right)=\frac{B(n-2)}{n-1} \\
P\left(t_{N W}\right)=\frac{B}{n-1} & P\left(t_{\beta}\right)=C & P\left(t_{S W}\right)=0 .
\end{array}
$$

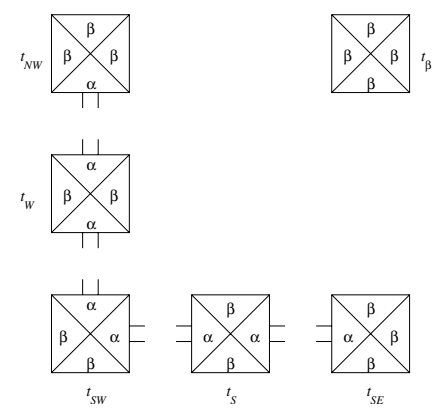

Fig. 2. The set of tiles used for producing rectangles

If we want to produce squares it is rather natural to create diagonals. Informally, we use three tiles in order to construct the diagonal. These three tiles $-t_{D}, t_{D_{\text {down }}}, t_{D_{\text {up }}}$ - appear in Figure 3. We need two more tiles in order to fill the square: $t_{\beta_{2}}$ for the northwest half and $t_{\beta_{1}}$ for the southeast half. We need to have 
$\beta_{2} \neq \beta_{1}$, in order to avoid final productions which are not squares. The seed is $t_{D}$ and the temperature is $\tau=2$.

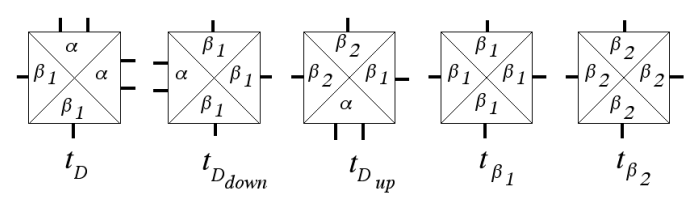

Fig. 3. The set of tiles used for producing squares

For arbitrary positive values $A, B, C, D$ such that $A+B+C+D=1$, we fix the concentrations as follows:

$P\left(t_{D}\right)=\frac{A(n-2)}{n-1}, P\left(t_{\beta_{1}}\right)=\frac{A}{n-1}, P\left(t_{D_{\text {down }}}\right)=B, P\left(t_{D_{u p}}\right)=C, P\left(t_{\beta_{2}}\right)=D$

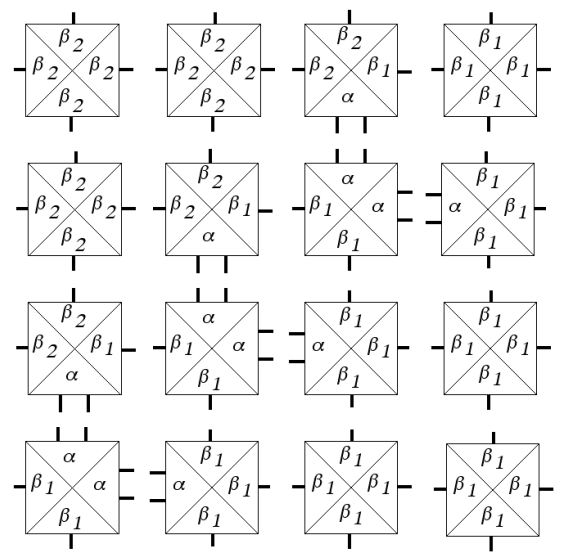

Fig. 4. An example of square production

Proposition 2. The tile systems defined above uniquely produce rectangles and squares. In the case of rectangles, if $M$ is the random variable corresponding to the width and $N$ the random variable corresponding to the height, then $\mathbb{E}(M)=m$ and $\mathbb{E}(N)=n$. In the case of squares, if $N$ is the random variable corresponding to the length of the side then $\mathbb{E}(N)=n$.

Proof. Just for $M$ in the case of rectangles. The other cases are very similar. Let $\epsilon=(m-1)^{-1}$. The random variable $M$ follows a geometric law. More precisely, $\operatorname{Pr}\{M=k\}=(1-\epsilon)^{k-2} \epsilon$. So $\mathbb{E}(M)=\epsilon^{-1}+1=m$. 
Proposition 3. The tile systems which we defined above are the smallest ones that uniquely produce the set of all rectangles and the set of all squares. In the case of rectangles, each set of tiles whose set of final productions is formed by the $m \times n$ rectangles, with $m \geq 2$ and $n \geq 2$, contains at least six tiles. In the case of squares, each set of tiles whose set of final productions is formed by the $n \times n$ squares, with $n \geq 2$, contains at least five tiles.

Proof. We just need to focus on glues of strength 2 . With respect to that property there are 16 tiles.

Squares. Let us consider the set of all possible tilings of $2 \times 2$ squares. We have 8 cases (see Figure 5). Since we are assuming the seed to be in the leftmost position of the bottom the number of cases can be reduced to 5 (up to symmetry $1=3,2=4,6=7)$. In each case, we have at least three or four tiles. More precisely, 2 of cardinality three and 3 of cardinality four.

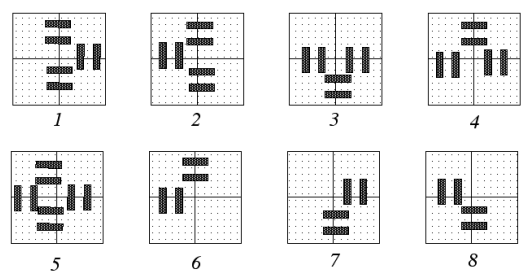

Fig. 5. The possible tilings of the $2 \times 2$ square

We want to prove that four tiles are not enough to generate squares. We have to test sets of four tiles which contain one of the previous sets which produce $2 \times 2$ squares. Each of the two cases of cardinality 3 may be completed with 13 tiles. Therefore, there are at most $2 \times 13+3=29$ cases to test. We first try to produce a $3 \times 3$ square with each set of tiles. In any exploration, we can easily see the impossibility for all the completions with the exception of the set numbered by 8 .

For this latter case, we study the only (hypothetical) possible tiling of a $4 \times 4$ square obtained by self-assembly. Let $t$ be the tile which has no glue of strength 2 . Notice that $t$ must be placed at the lower right corner and at the upper left corner of the square. Moreover, the opposite glues of $t$ must be equal (in order to assemble any $k$-square with $k>2$ ). Let $t^{\prime}$ be the tile with glue of strength 2 in its southern side. A $t$-tile of the left upper part of the square must touch a $t^{\prime}$-tile below and another $t$-tile must touch a $t^{\prime}$-tile in its right. Therefore, a $t^{\prime}$-tile could be placed in the lower right corner of the square. A contradiction.

Rectangles. For rectangles we consider set of tiles which can produce the $2 \times 2$ square (i.e. containing a set of tiles obtained in Figure 5), the $3 \times 2$ rectangle, $2 \times 3$ rectangle.

We first assume that the only glue of strength 2 of the seed is on its northern side. In this case, we have 12 possible tilings of the $3 \times 2$ rectangle induced by 
successive productions, 7 with six tiles, 4 with five tiles, and 1 with four tiles. An obvious analysis proves that sets of 5 tiles obtained are not sufficient to tile the $2 \times 3$ rectangle.

For the set of four tiles, we can remark that another tile is necessary to tile the $2 \times 3$ rectangle and, furthermore, there exists two tiles with only a glue of strength 2 on their northern side which have the same strength 2 glue. Otherwise, some productions which are not in the upper right quarter of plane appear.

The case when the only glue of strength 2 of the seed is on its eastern side can be treated in a symmetric way. We now assume that the seed has two sides (the northern and the eastern one) with glues of strength 2. We study tilings of the $3 \times 2$ rectangle induced by successive productions We have two subcases according to the glues of strength 2 of the tile in position $(0,1)$.

- When this tile has two sides (the southern and the eastern one) with glues of strength 2 , we also have 12 cases: 7 with six tiles, 4 with five tiles, and 1 with four tiles. An obvious analysis proves that the sets of 5 tiles obtained are not sufficient to tile the $2 \times 3$ rectangle.

- When this tile has only one side (the southern one) with a glue of strength 2 , we also have 12 cases (symmetric to the case when the seed has a unique side with glue of strength 2): 7 with six tiles, 4 with five tiles, and 1 with four tiles. An obvious analysis proves that the sets of 5 tiles obtained are not sufficient to tile the $2 \times 3$ rectangle.

Thus, we have a tricky case only when there exists a set of five tiles, such that four of them are enough to tile the $3 \times 2$ rectangle, and four of them are enough to tile the $2 \times 3$ rectangle (see figure 6). We have (up to symmetry) only one set of five tiles satisfying this condition. But, according to the number of glues of strength 2, this set either has final productions which are not rectangles, or does not produce all rectangles. This finishes the proof.
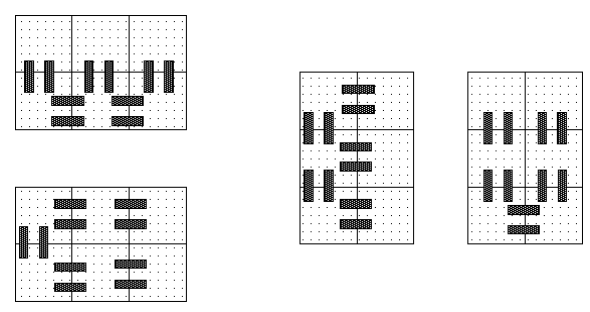

Fig. 6. The possible tilings with four tiles for the $3 \times 2$ rectangle and the $2 \times 3$ rectangle, with a seed with two glues of strength 2 . A union of two tiling sets (one for each rectangle) contains at least 5 tiles. Moreover, the only set (up to symmetry) of 5 tiles obtained by this way is formed by tiles used in the highest $3 \times 2$ rectangle and in the rightmost $2 \times 3$ rectangle. 
Proposition 4. With the tile systems defined above, the parallel time needed to assemble an $m \times n$ rectangle is $m+n$ while the parallel time needed to assemble an $n \times n$ square is $3 n-5$.

Proof. We give a proof for the squares (rectangles are similar). Notice first that for any shape $S$ with a marked position $(x, y)$, the parallel time to assemble $S$ with the seed at $(x, y)$ is at least $\max _{\left(x^{\prime}, y^{\prime}\right) \in S}\left\{d\left((x, y),\left(x^{\prime}, y^{\prime}\right)\right\}\right.$, no matter which tileset is used. The parallel time needed to put the tile at $(n-1, n)$ is at least $2 n-3$ since that is the distance $l_{1}$ between $(0,0)$ and $(n, n-1)$. This tile is the only one in the line $\{(x, n), 0 \leq x \leq n\}$ with a glue of strength 2 on its south side, thus it has to be put before any other in this line. Thus, the tile at $(n, 0)$ cannot be put before the step $2 n-3+n-2=3 n-5$. It is then easy to see that this bound is in fact reached, and that the square can be assembled in time $3 n-5$.

\section{Diamonds}

Notice that a square corresponds to the set $\left\{(x, y) \mid d_{\infty}[(x, y),(0,0)] \leq n\right\}$, with $d_{\infty}\left[(x, y),\left(x^{\prime}, y^{\prime}\right)\right]=\max \left\{\left|x-x^{\prime}\right|,\left|y-y^{\prime}\right|\right\}$. If we change the metrics towards the more "natural" $d_{1}\left[(x, y),\left(x^{\prime}, y^{\prime}\right)\right]=\left|x-x^{\prime}\right|+\left|y-y^{\prime}\right|$, then the induced shape is the diamond $D_{n}$ that appears in Figure 7. The problem of producing diamonds is much more complicated than those we tackled before. Any naive approach seem not to work.

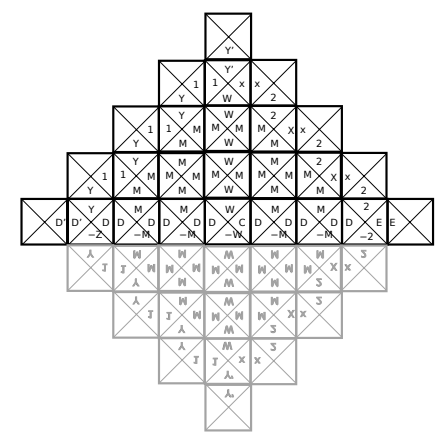

Fig. 7. An n-diamond

A first possible approach is given by the tileset of Figure $8(\mathrm{a})$ This tileset assembles the upper halves of diamonds. By using this set and its mirror image, one can assemble the set of all diamonds. The tileset works by "knitting" the halfdiamond row after row, going back and forth (see Figure $8(\mathrm{~b})$. The sequentiality of this approach is the cause of the quadratic parallel time $n^{2} / 4$. We are going to show how to lower this bound. More precisely, we are going to construct diamonds in linear parallel time with the help of a very particular and nontrivial cellular automaton called "the firing squad". 


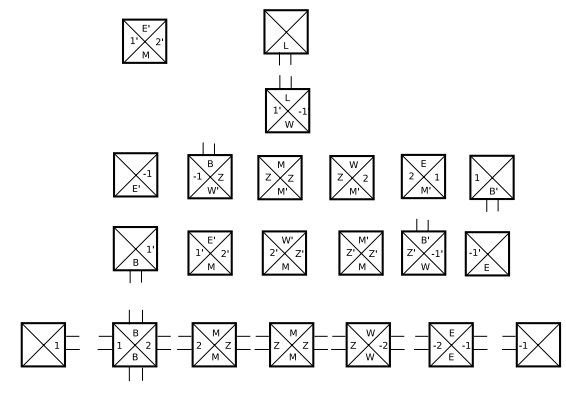

(a) The tileset for assembling halfdiamonds

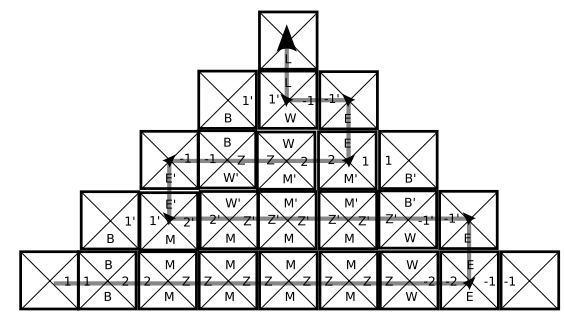

(b) The assembly of a half-diamond

Fig. 8. Knitting diamonds

\subsection{Simulating the Firing Squad CA by Self-assembly}

The firing squad automaton, detailed in [3, is a cellular automaton which, from a line of $n$ cells, such that the first and $n$-th cells are in special states $G_{l}$ and $G_{r}$, and the other in initial state $s$, evolves in such a way that they all enter the final state $F$ for the first time at time $n$, and all other cells remain in a quiescent state $\rho$.

We will represent, for simplicity, the tiles rotated in $45^{\circ}$. Let us first consider the set of six tiles of Figure 9. The tiles are, from left to right, $t_{\alpha}$ (the seed), $t_{G_{l}}, t_{\beta}, t_{s}, t_{G_{r}}$ and $t_{\gamma}$. The colors $\alpha, \beta, \gamma \notin Q_{F S}$. The color null is omitted. For instance, $\sigma_{N}\left(t_{\alpha}\right)=\sigma_{S}\left(t_{\alpha}\right)=\sigma_{W}\left(t_{\alpha}\right)=$ null.

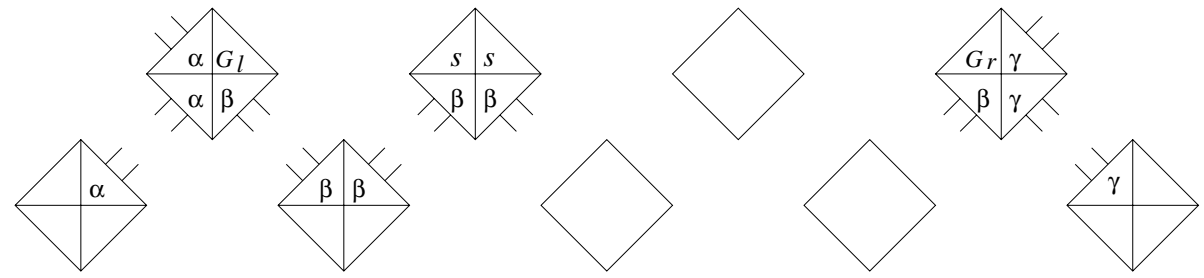

Fig. 9. The set of six tiles that codifies the initial configuration

As it is schematically explained in Figure 10, the assemblying process of these tiles is such that the structure they produce represents the initial configuration $\ldots \rho \rho \rho G_{l} s s s \ldots s s s G_{r} \rho \rho \rho \ldots$.

The size of the initial structure of Figure 10 determines the size of the diamond the tile system is going to produce. This part of the self-assembly process is, in fact, the only nondeterministic one. Therefore, the expected size of the diamond can be calculated as a function of the concentrations of the previously introduced 


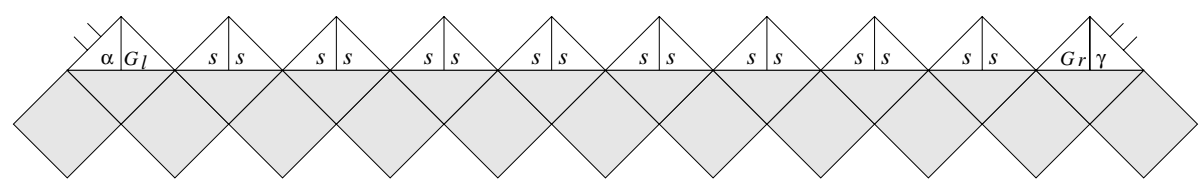

Fig. 10. The way the initial configuration $\ldots \rho \rho \rho G_{l} s s s \ldots s s s G_{r} \rho \rho \rho \ldots$ is assembled

tiles. Moreover, the only concentrations relevant for the process are those of $t_{s}$ and $t_{G_{r}}$. Let $0<A<1$. Let us define the concentrations as follows:

$$
P\left(t_{s}\right)=A\left(1-(n-1)^{-1}\right), P\left(t_{G_{r}}\right)=A(n-1)^{-1} .
$$

The only requirement for the concentrations of the other tiles (the four already introduced and those to come) is that they must be positives with their sum being $1-A$.

There are two other classes of tiles: transmission tiles and transition tiles. The transmission tiles are divided into six subclasses: left-border, internal, rightborder, upper-left-border, upper-border, upper-right-border. More precisely, for all $a, b \in Q_{F S} \backslash\{F\}$, the transmission tiles are constructed in Figure [1].
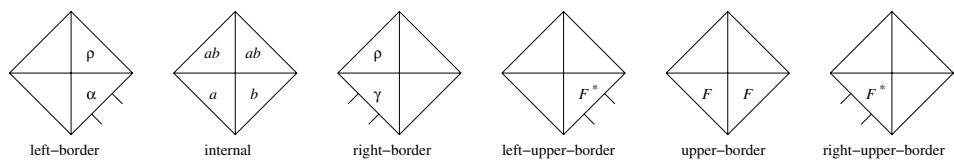

Fig. 11. Transmission tiles

The transition tiles are divided into five subclasses: left-border, internal, rightborder, upper-left-border and upper-right-border.

More precisely: let $a, b, c, d, e, f, g \in Q_{F S}$ be such that $\delta_{F S}(\rho, a, b) \neq F$ and $\delta_{F S}(f, g, \rho) \neq F$. The $(a, b)$-left-border, $(c, d, e)$-internal and $(f, g)$-right-border tiles are constructed in Figure $12(\mathrm{a})$.

Finally, let $a, b, c, d \in Q_{F S}$ be such that $\delta_{F S}(\rho, a, b)=\delta_{F S}(c, d, \rho)=F$. The upper-left-border and upper-right-border tiles are constructed in Figure 12(b)

From the previously defined construction follows the last propositions.
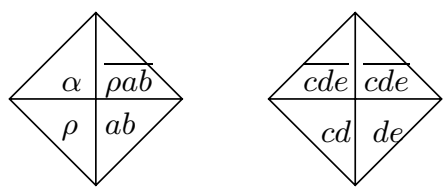

(a) Left-border, internal, right-border.
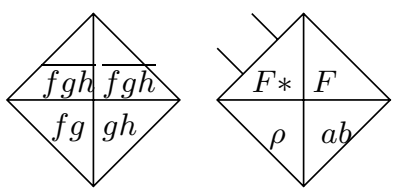

(b) Upper-left-border, upperright-border.

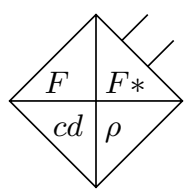
(1)

Fig. 12. Transition tiles; $\overline{x y z}$ represents $\delta_{F S}(x y z)$ 
Proposition 5. The tile system defined above uniquely produces diamonds. If $N$ is the random variable corresponding to the length of the diagonal, then $\mathbb{E}(N)=$ $2 n+1$; and the parallel time needed by the above tile system to assemble a diamond of size $n$ is $4 n-6$.

Proof. The parallel assembly follows the simulation of the $C A$, as shown on Figure 13. By induction one gets that the last tile of the $k$ th row is added in the $2 n+k-4$ parallel transition for $k>0$. As the assembly is complete when the $(2 n-2)$ th row of the simulation is complete, the parallel time for the assembly is $4 n-6$.

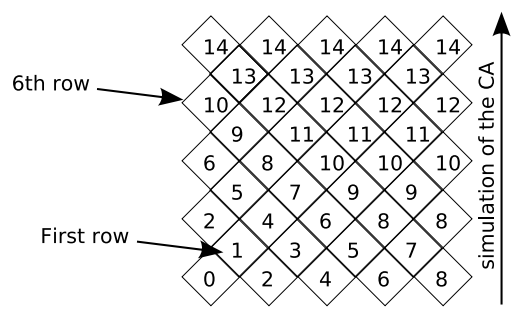

Fig. 13. Parallel assembly of a diamond

\section{References}

1. L. Adleman, Q. Cheng, A. Goel and M. Huang. "Running time and program size for self-assembled squares". In Proceedings of the 33rd Annual ACM Symposium on Theory of Computing (STOC 2001), 740-748, 2001.

2. G. Aggarwal, M. H. Goldwasser, M-Y. Kao and R. T. Schweller. "Complexities for generalized models of self-assembly". Proceedings of the 15th Annual ACM-SIAM Symposium on Discrete Algorithms (SODA 2004), 880-889, 2004.

3. J. Mazoyer and N. Reimen. "A linear speed-up theorem for cellular automata". Theoretical Computer Science 101 (1992), 59-98.

4. P. Rothemund . "Theory and experiments in algorithmic self-assembly". Ph. D. Thesis, University of Southern California, 2001

5. P. Rothemund and E. Winfree. "The program size complextity of self-assembled squares". In Proceedings of the 32nd Annual ACM Symposium on Theory of Computing (STOC 2000), 459-468, 2000.

6. H. Wang. "Proving theorems by pattern recognition". H. Bell System Technical Journal, 40:1-41, 1961.

7. E. Winfree. "Algorithmic self-assembly of DNA". Ph.D. thesis, California Institute of Technology, Pasadena, 1998. 